\title{
Evolution du vivant - Histoire du pensant De la possibilité d'un homomorphisme
}

\author{
par Gérard Donnadieu \\ Vice-Président de l'AFSCET \\ Professeur de sciences religieuses au Collège des Bernardins et aux Facultés jésuites de Paris \\ gerard.donnadieu@wanadoo.fr
}

\begin{abstract}
Résumé : La recherche de similitudes ou de corrélations entre l'évolution des êtres vivants et celle des sociétés humaines n'est pas chose nouvelle. Les philosophes s'y sont longtemps essayés avant que les scientifiques ne prennent le relai. En tirant partie des nouvelles découvertes survenues dans ce dernier demi-siècle tant dans les sciences de la vie que dans les sciences sociales, est-il possible de reprendre aujourd'hui cette réflexion à nouveaux frais? Telle est l'ambition de la présente communication qui s'efforce d'éclairer l'une par l'autre ces nouvelles découvertes faites sur le vivant et le pensant en les fédérant au moyen d'un concept emprunté à l'approche systémique : l'homomorphisme. A mi-chemin de la métaphore et de la modélisation à prétention exhaustive, l'homomorphisme est une analogie partielle et imparfaite entre deux systèmes concrets, analogie néanmoins éclairante et féconde.

Cet homomorphisme sera mené à un double niveau :

- celui des moteurs de l'évolution du vivant d'une part, en reprenant des travaux récents qui cherchent à dépasser le néo-darwinisme; des moteurs de l'histoire humaine d'autre part, à partir des analyses du grand historien britannique Arnold Toynbee.

- celui de la description phénoménologique de l'évolution sur la longue durée, aussi bien celle des systèmes vivants que des cultures humaines et civilisations. Peut-on discerner dans cette "évolution longue" des lois tendancielles et y découvrir une orientation (au double sens de direction et de signification) comme le pensait Teilhard de Chardin?

Par ces confrontations, l'auteur de la présente communication espère montrer que l'évolution du vivant peut aider à comprendre l'histoire du pensant, et réciproquement que l'histoire des cultures humaines et des civilisations peut aider à comprendre la plasticité et l'inventivité du vivant.
\end{abstract}

Mots clefs : analogie, civilisation, culture, défi-réponse, évolution, histoire, homomorphisme, mutation, sélection naturelle, symbiose, union créatrice.

Abstract : The search for similarities or correlations between the evolution of living beings and human societies is nothing new. Philosophers have tried to answer the problem for a long time, before leaving it to scientists. Considering the new discoveries made during the latest half-century in the science of the living as well as in social science, is it possible to undertake this study in a new way? This is the aim of the present communication, it seeks to enlighten one on the other the new discoveries made on the living and on the thinking by federating them thanks to a concept belonging to the systemic approach: homomorphism. Half-way between a metaphor and the supposedly exhaustive setting-up of a model, homomorphism is a partial and imperfect analogy between two concrete systems, an analogy able to give light and be fruitful at the same time.

This homomorphism will be looked for on two levels:

- the level of the driving force behind the evolution of the living on the one hand, by reverting to the recent works that aim to go beyond neo-darwinism; of the driving force behind human history on the other hand, starting from the analysis of the great British historian Arnold Toynbee,

- the level of the phenomenological description of evolution over the long term, regarding living systems as well as human cultures and civilizations. Can one discover rules in this "long evolution" tendency and discover there a twofold orientation (meaning both direction and meaning) as Teilhard de Chardin thought?

Thanks to these confrontations, the author of the present paper hopes he can show that the evolution of the living can help understand the history of the thinking, and reciprocally that the history of human cultures and civilizations can help understand the plasticity and inventiveness of the living.

Key words : analogy, civilization, culture, answer-challenge, evolution, history, homomorphism, mutation, natural selection, symbiosis, creative union. 
On sait que l'approche systémique a restauré la valeur du raisonnement analogique dans le discours scientifique. Largement connu et utilisé par les philosophes de l'Antiquité et les théologiens médiévaux, ce mode de raisonnement s'est trouvé décrié au $19^{\text {ème }}$ siècle au nom d'un rationalisme étroit...alors même qu'il continuait d'imprégner la démarche heuristique des chercheurs. Ces derniers n'hésitent pas en effet à transposer à leur domaine de manière "sauvage" des concepts venus d'autres disciplines, quitte à le justifier ensuite par de laborieux raisonnements déductifs. Transposition heureuse, comme le souligne Edgar Morin ${ }^{1}$ : "La circulation clandestine des concepts a permis aux disciplines de se désasphyxier".

Trois niveaux d'analogie peuvent ainsi être distingués :

- la métaphore qui établit une correspondance souvent toute extérieure entre deux systèmes de nature différente. Parce qu'elle se fonde sur l'apparence, la métaphore est dangereuse et tombe sous le coup de l'adage positiviste "comparaison n'est pas raison". Mais bien utilisée, la métaphore est néanmoins précieuse. En stimulant l'imagination, elle facilite la création de nouveaux modèles et constitue souvent un premier pas vers l'homomorphisme,

- l'homomorphisme établit une correspondance surjective entre quelques traits du système étudié et les traits d'un modèle théorique ou d'un système concret plus simple ou plus commodément étudiable (que l'on appelle alors modèle réduit). Par des observations effectuées sur le second système, il devient alors possible de prévoir certains aspects du comportement du premier,

- l'isomorphisme est la seule analogie acceptable dans une démarche analytique de type cartésien. Il s'agit d'établir une correspondance bijective entre tous les traits du système étudié et ceux d'un modèle censé reproduire une image "parfaite" de l'original où rien n'est oublié, ni dans la description, ni dans le fonctionnement.

Utilisable pour les systèmes à faible complexité de la physique et de la chimie, l'isomorphisme n'est guère tenable pour les systèmes plus complexes du vivant et du social, et cela malgré la tentation d'augmenter considérablement les variables prises en compte. Par un glissement inévitable, on en est donc venu à accepter l'homomorphisme, c'est-à-dire l'imperfection du modèle, comme un mal nécessaire et utile. Le modèle homomorphe est sans doute plus simple que le réel, mais c'est pourquoi nous le comprenons, pourquoi nous pouvons l'utiliser pour orienter nos actions. Comme le note Claude Lévi-Strauss ${ }^{2}$. "La vertu intrinsèque du modèle réduit est qu'il compense la renonciation à des dimensions sensibles par l'acquisition de dimensions intelligibles". Et ceci est particulièrement heureux car le but d'un modèle n'est pas l'exhaustivité. Il est des détails inutiles pour le but visé, que ce but soit la construction de modèles pour comprendre (qui doivent être suffisamment simples) ou de modèles pour agir (qui doivent être opératoires).

C'est un tel homomorphisme que nous souhaitons mettre en œuvre, à propos du concept d'évolution, entre les systèmes vivants produits dans la nature depuis 3,5 milliards d'années et les systèmes pensants, c'est-à-dire l'homme et les sociétés humaines dont l'origine ne remonte guère à plus de cent mille ans. Quelles similitudes peut-on établir entre l'évolution du vivant (ou biogénèse) elle-même prolongement de l'évolution du cosmos (ou cosmogénèse) depuis le big-bang il y a 13,8 milliards d'années et l'évolution de l'homme et de l'humanité, appelée plus communément Histoire et dont le philosophe Michel Serres nous dit qu'il s'agit de ce qu'il y a de "plus fortement complexe" dans le monde où nous vivons ?

Une telle recherche de similitudes ou de corrélations entre l'évolution des êtres vivants et celle des sociétés humaines n'est pas chose nouvelle. Les philosophes s'y sont longtemps essayé avant que les scientifiques ne prennent le relai, non sans difficultés d'ailleurs comme

\footnotetext{
1 MORIN Edgar, Introduction à la pensée complexe, p. 154, ESF, 1991

${ }^{2}$ LEVI-STRAUSS C. : La pensée sauvage, Plon, 1962
} 
le montre l'histoire de la très discutable sociobiologie. Mais nous pensons que le temps est venu où il est enfin possible de reprendre cette réflexion à nouveaux frais. Nous le ferons à un double niveau à partir d'une distinction classique établie par le biologiste Michel Morange, professeur à l'Ecole Normale Supérieure de Paris :

- le niveau du moteur de l'évolution du vivant d'une part, en reprenant des travaux récents qui cherchent à dépasser le néo-darwinisme; du moteur de l'histoire humaine d'autre part. Par exemple, s'agissant du vivant, quelle importance donner au mécanisme mutationsélection naturelle de la théorie dite synthétique ou à celui de symbiose créatrice dans les théories plus récentes ? Et concernant l'histoire des civilisations, quelle place reconnaitre au mécanisme défi-réponse du grand historien britannique Arnold Toynbee ?

- le niveau de la description phénoménologique de l'évolution sur la longue durée, aussi bien celle des systèmes vivants que des cultures humaines et civilisations. Peut-on discerner dans cette "évolution longue" des lois tendancielles et y découvrir une orientation (au double sens de direction et de signification) comme le pensait Teilhard de Chardin?

\section{Quel moteur pour l'évolution du vivant ?}

Voici 40 ans, lorsque Jacques Monod écrivait Le hasard et la nécessité, on pouvait croire le problème du moteur de l'évolution résolu. Dans la théorie synthétique (ou néodarwinienne) de l'évolution, telle qu'elle fut théorisée par le biologiste Ernst Mayr (19052005) dans les années 1950, tout paraît en effet s'expliquer par deux facteurs seulement : le hasard des mutations génétiques qui est une source inépuisable de diversité biologique ; la sélection naturelle exercée par l'environnement et qui ne laisse subsister que les individus ou les espèces vivantes les plus aptes. Pour que sur la base d'une mutation génétique une population donne naissance à une nouvelle espèce, il faut alors que le mutant se sépare de l'espèce souche pour constituer un isolat. Par effets cumulatifs jouant sur la longue durée, on pense pouvoir obtenir ainsi l'extraordinaire variété du monde vivant, mais sans que l'on puisse dire qu'une direction particulière ait été privilégiée par l'évolution, celle-ci s'étant effectuée dans toutes les directions à la fois selon un processus buissonnant. C'est cette théorie qui reste encore dominante dans l'enseignement officiel des lycées français.

\section{1-1) La crise de la théorie synthétique}

Et pourtant, comme l'ont montré les nombreux colloques et débats qui se sont déroulés en 2009 lors de l'année Darwin, les choses apparaissent aujourd'hui singulièrement plus complexes. De nombreux autres mécanismes susceptibles d'influer sur le processus évolutif ont été identifiés par les chercheurs. Citons, sans prétendre être complet :

- la théorie des équilibres ponctués de Stephen Jay Gould. Contre le gradualisme prêté à l'évolution par Darwin et la théorie synthétique, celle-ci procéderait par sauts, faisant succéder de brèves périodes d'emballement à de longues périodes de stabilité (dites stases)

- la théorie de la convergence fonctionnelle du paléontologue britannique Simon Conway Morris. Pour lui, les "inventions" du vivant sont partiellement prévisibles et se produisent dès que des conditions favorables sont à peu près réalisées dans la nature ; elles ne s'expliquent donc par le seul hasard! Ainsi, un organe comme l'œil des vertébrés, aussi merveilleusement conçu pour la vision et fabuleusement complexe, va émerger au moins à trois reprises au cours de l'évolution avant de l'être chez les poissons à partir desquels cet organe sera définitivement transmis à tous les vertébrés. Il y aurait donc dans la nature une évolution spontanée vers la haute cognition qui culmine au niveau humain mais que l'on retrouve en ébauche dans bien d'autres lignées animales. 
- l'interaction systémique environnement/espèces ou co-évolution. Cette théorie met en évidence l'importance des boucles de rétroaction existant entre un environnement et des espèces qui bougent en permanence. Et ces boucles, extrêmement nombreuses, peuvent être de polarité négative, positive ou ago-antagoniste. Nous voici là au cœur de l'hypercomplexité dont on sait combien elle favorise les effets d'émergence.

- la théorie évo-dévo (de l'anglais evolutionary developmental biology), point de rencontre, selon Michel Morange, entre la biologie fonctionnelle et la biologie de l'évolution. Elle cherche en effet à concilier l'approche évolutive et le contrôle génétique du développement d'un organisme, tel qu'il s'observe par exemple en embryologie. L'évolution apparaît dans ce cas comme canalisée et orientée sur des bases internes.

- le rôle possible de comportements pouvant être transmis par apprentissage, notamment chez les animaux supérieurs. Ces comportements vont favoriser ensuite le développement ou l'inhibition de certains organes et se traduire au final par des modifications somatiques un peu à la manière dont l'envisageait Lamarck. La conséquence peut en être un raccourcissement considérable des temps requis pour les transformations d'espèces.

Le débat autour du moteur de l'évolution est donc aujourd'hui particulièrement ouvert et l'on semble enfin sorti du simplisme réducteur de la théorie synthétique.

\section{1-2) Un paradigme émergent : I'UNION CREATRICE}

Ce terme apparait pour la première fois en pleine guerre de 14-18 dans l'œuvre du paléontologue jésuite Pierre Teilhard de Chardin, à l'occasion d'un court essai ${ }^{3}$ adressé à sa cousine Marguerite. Par la suite, Teilhard reviendra à plusieurs reprises sur cette notion à l'occasion de ses travaux de paléontologie humaine, jusqu'à rédiger en 1944, à la fin de son séjour à Pékin, un nouvel essai ${ }^{4}$ plus ambitieux et de facture plus rigoureuse. Il y montre comment l'union complexifiante de composants organiques déjà préexistants fait émerger un centre, lequel se comporte comme facteur d'unité et de finalisation de l'ensemble ainsi créé. Cette union est donc créatrice car elle donne un intérieur, un "en soi", une "conscience" en quelque sorte, à ce rassemblement organisé de composants. C'est pourquoi la montée en complexité des systèmes vivants s'accompagne de l'apparition de psychismes de plus en plus riches, ce que Teilhard exprime par sa loi de complexité-conscience.

En restant au niveau du vivant, l'union créatrice met ainsi l'accent sur l'importance des symbioses qui sont le plus souvent à la base des grandes inventions de la vie. Par exemple le passage de la bactérie à la cellule eucaryote qui prendra plus d'un milliard d'années et résulte de la mise en symbiose de plusieurs bactéries; l'apparition des métazoaires (plantes et animaux) qui résulte de l'association organique de plusieurs cellules ; l'invention de la sexuation il y a 800 millions d'années, laquelle repose sur l'union des sexes et s'avère un merveilleux générateur de diversité en matière de reproduction ; l'invention de la structure tridermique des vertébrés par rapport à celle des mollusques qui rendra possible l'émergence d'un système nerveux central; l'apparition bien sûr de la pensée réfléchie à partir de la mise en réseau de quelques gros cerveaux d'homo sapiens.

Le spécialiste de biologie végétale, Jean-Marie Pelt, a mis en évidence dans plusieurs ouvrages $^{5}$ l'importance de ces symbioses tant au niveau animal que végétal. Un autre biologiste, Pierre Bricage ${ }^{6}$, a cherché à en construire un modèle théorique qu'il a baptisé

\footnotetext{
${ }^{3}$ Cet essai figure dans les Ecrits du Temps de la guerre, pp.194-224, Tome 12 des Euvres complètes, Seuil

${ }^{4}$ Pierre TeILHARD DE CHARDin, La Centrologie. Essai d'une dialectique de l'Union, L'Activation de l'Energie, pp.122-124, Tome 7 des Euvres complètes

5 Jean-Marie PELT, La raison du plus faible, Fayard, 2009 ; Le monde a-t-il un sens, Fayard, 2014

${ }^{6}$ Pierre BRICAGE, L'approche systémique de l'évolution du vivant, Teilhard Aujourd'hui n`33, mars 2010. Dans un article antérieur publié en septembre 2005 dans la revue informatique Res-Systemica et intitulé "L'origine
} 
ARMSADA (Associations for the Reciprocal and Mutual Sharing of Advantages and of Disadvantages ou en français "Associations à avantages et inconvénients réciproques et partagés"). Il y montre comment l'union durable de plusieurs espèces qui en viennent à mettre en commun leurs génomes finit par faire émerger une nouvelle espèce plus complexe, de niveau d'organisation supérieur et dotée de propriétés absolument nouvelles.

\section{Et quel moteur pour l'Histoire de l'Humanité ?}

Dans le monde si divers des historiens, le britannique Arnold Toynbee (1889-1975) se présente comme l'un des rares à vouloir penser "l'histoire totale" de l'humanité. Au terme d'une vie entière consacrée à sa discipline, il nous invite à considérer l'histoire d'un point de vue extrêmement élevé, le seul d'où ses lignes de force apparaissent avec une remarquable clarté. Cette prétention explique le curieux mélange d'admiration et d'indignation dont son œuvre est l'objet parmi les historiens.

Quelle est la méthode d'Arnold Toynbee dans sa démarche historienne d'intention scientifique, quelle est la grille d'interprétation qu'il se propose de jeter sur le réel ${ }^{7}$ ?

Pour Toynbee, l'historien a pour mission, à l'instar de tout scientifique, de rendre intelligible (ou compréhensible) le foisonnement de l'observable. Mais pour lui, cette explication ne peut reposer sur le dévoilement de quelque variable cachée : le sens de l'Histoire du marxisme, le développement de l'Esprit universel de Hegel, le doigt de Dieu ou la divine Providence des théodicées. Les lois de l'histoire sont à rechercher dans l'histoire ellemême. En quelque sorte, l'histoire doit expliquer l'histoire.

Pour cela, Toynbee va chercher à mettre en évidence dans l'histoire de l'humanité, des champs d'observation relativement autonomes, des correspondances d'évolution, des régularités de fonctionnement.

\section{2-1) La civilisation comme unité de champ d'intelligibilité}

Contrairement à l'historiographie traditionnelle (thèse dominante tout au long du 19 ème siècle notamment), la nation ne constitue pas pour Arnold Toynbee l'unité pertinente d'observation historique. Même pour des nations aussi anciennes que la France et l'Angleterre, il disqualifie cette prétention en montrant le dense réseau de leurs influences et emprunts réciproques. Pour lui, les champs intelligibles de l'étude de l'histoire "sont des sociétés qui ont une plus grande expansion, dans le temps et dans l'espace, que des nations ou des citésEtats ou que n'importe quelles autres communautés politiques".

Ces unités de champ intelligible, qui émergent à l'aube des temps historiques, il les désigne sous le nom de civilisations. Rendues possibles par la grande mutation néolithique d'il y a dix mille ans (invention de l'agriculture et de l'élevage), les civilisations sont liées à la fondation d'une cité ou d'une ville. Grâce à la première division du travail, la ville permet en effet à une fraction de la population de se libérer de l'activité économique immédiate et de développer une activité exclusive dans l'ordre de la pensée. Cette "caste sacerdotale" fait émerger au sein de la société concernée une certaine vision de l'organisation à mettre en place, des rapports sociaux à conserver ou à créer, de l'harmonie à maintenir. C'est tout cela que Toynbee appelle une "civilisation". Elle constitue pour lui "un champ intelligible d'études historiques" comme système spécifique de relations entre un certain nombre d'individus, de groupes et peuples différents. La civilisation serait donc la véritable "unité organique" depuis

endosyncénotique de la cellule" - (http://www.afscet.asso.fr/resSystemica/Paris05/bricage3.pdf) -Pierre Bricage donnait une présentation complète de son modèle. Voir aussi le site http://armsada.eu .

${ }^{7}$ Elles sont développées dans son œuvre monumentale en 12 volumes A Study of History, puis résumées dans son ouvrage de synthèse L'Histoire, Elsevier, 1978 
l'entrée dans la période historique (en gros, depuis 5000 ans) ; les nations ou cités-Etats n'étant que des parties ou sous-systèmes de cet ensemble cohérent.

Toynbee observe alors qu'aucune société particulière érigée en civilisation n'a encore réussi à embrasser l'ensemble de l'humanité dans l'espace et dans le temps, s'étendant à ce que Teilhard de Chardin appelle la noosphère. Et il décompte la succession de 34 civilisations depuis l'origine des temps historiques, la plupart ayant disparu et cinq restant encore au milieu du $20^{\text {ème }}$ siècle des "affaires qui marchent". Enfin, bien que chacune de ces civilisations puisse être considérée comme une entité homogène et autonome, il existe toujours des échanges et des filiations de l'une à l'autre ; il s'agit de "systèmes ouverts".

Pour Toynbee, les civilisations les plus récentes sont caractérisées par ce qu'il appelle des religions supérieures dont "le trait distinctif par rapport aux religions de type antérieur, est qu'elles s'adressent directement aux hommes comme à des personnes individuelles, et non par l'intermédiaire des sociétés au sein desquelles ces personnes se livrent à leurs autres activités". Alors que dans les sociétés primitives ou simplement traditionnelles, le social et le religieux sont indissolublement mêlés (ce qui est la thèse du sociologue Emile Durkheim reprise aujourd'hui par Marcel Gauchet ${ }^{8}$ ), les religions supérieures "ont perçu que le salut doit être recherché, non dans le domaine des relations sociales, mais dans le domaine de la vie spirituelle de la personne". Toynbee décompte ainsi six religions supérieures : hindouisme, zoroastrisme, judaïsme, bouddhisme, christianisme, islam, les trois dernières étant plus universelles que les trois premières qui restent davantage liées à leur matrice ethnique d'origine. En pratique cependant, ces religions supérieures sont obligées de composer avec les religions de la nature et de la société qui les ont précédées et ne réussissent à produire leurs fruits spirituels que parmi une élite de disciples (par exemple les moines et moniales dans le bouddhisme). Et ce n'est que très progressivement que se réalise, dans les comportements collectifs, la distinction claire entre le spirituel et le social ${ }^{9}$.

\section{2-2) Les processus de croissance des civilisations}

L'observation historique montre qu'une nouvelle civilisation naît soit d'une mutation émergente à partir d'une société primitive, soit d'une crise profonde remaniant de l'intérieur une civilisation plus ancienne. Mais quels en sont les mécanismes et les conditions ?

a) Le paradigme du défi/réponse : Il s'agit là de la thèse centrale de Toynbee, lequel rompt avec la conception traditionnelle cause/effet pour lui substituer un concept de nature systémique, le défi/réponse dont il dit : "A la différence de l'effet d'une cause, la réponse à un défi n'est pas prédéterminée, n'est pas nécessairement uniforme et, par conséquent, est en soi imprévisible".

Le défi/réponse ouvre en effet sur la liberté et la créativité de l'acteur dont la réaction ne peut absolument pas être prédite mais jaillit, telle une nouvelle création, de la rencontre avec le défi lui-même. Et cette réaction ne peut se comprendre si l'on fait abstraction de la liberté de l'acteur, de son projet, de ses valeurs. De plus, le défi concernant une société, la réponse doit être aussi de nature collective et reposer sur le processus d'union créatrice de Teilhard jouant ici non pas à partir d'éléments biologiques mais d'acteurs humains intelligents et libres. Rapporté au modèle néo-darwinien de l'évolution, le défi représente en quelque sorte l'aiguillon de la nécessité et à cet aiguillon répond une réponse des acteurs humains sous forme d'une invention qui ne peut jamais être assimilée à une mutation due au seul hasard, même si ce hasard est utilisé dans le cadre d'un tâtonnement orienté. On notera que ce cycle

\footnotetext{
${ }^{8}$ Son ouvrage Marcel GAUCHET, Le désenchantement du monde, Gallimard, Paris, 1985

${ }^{9}$ Pour le socio-anthropologue Marcel GAUCHET, cette évolution n'a vraiment eu lieu que dans des sociétés marquées historiquement par le christianisme. On reconnaît là sa thèse sur "le christianisme comme religion de la sortie de la religion".
} 
adaptatif invention / nécessité est bien connu de l'histoire des techniques où il a de nombreuses illustrations.

Ainsi, par-delà le causalisme de la science positiviste, se trouve réintroduit dans la démarche scientifique le principe de finalité.

b) Quels défis ? Très souvent, pour une société encore néolithique, le défi a été de nature agro-technique (défrichement d'une terre, assèchement de marais, maîtrise de l'eau). Mais il a pu revêtir d'autres formes : aridité du sol de l'Attique pour Athènes, absence de cordon littoral agricole pour les Phéniciens obligés de ce fait à devenir marins, arrière-pays dangereux pour Byzance qui a installé ses habitants en "douaniers" des échanges Est/Ouest. Avec l'avènement des royaumes et des empires, le défi prend souvent une forme organisationnelle et appelle des réponses de nature politique, administrative, militaire

Mais si le défi est nécessaire pour faire naître une civilisation puis la pousser à se développer, il ne doit pas être excessif. Trop faible, le défi ne stimule pas, trop fort il écrase. Il y a en quelque sorte un niveau optimal du défi qui rend la réponse créatrice. Ainsi, la confrontation du christianisme avec l'islam a représenté, selon Toynbee, un stimulant :

- trop faible pour la chrétienté monophysite d'Abyssinie protégée par ses montagnes et ses déserts et qui est demeurée somnolente,

- trop fort pour la chrétienté orthodoxe du Moyen Orient qui a été asservie,

- optimal pour la chrétienté médiévale européenne qui a résisté et a donné naissance à la civilisation occidentale

c) L'entretien de la croissance : La croissance d'une civilisation est fondée sur une succession réussie de défis/réponses. Cette succession est toujours déclenchée par le premier défi I réponse, le plus difficile à surmonter et qui servira ensuite de référence et de modèle.

Ces défis sont relevés par les individus créateurs de la société, lesquels constituent toujours une élite minoritaire. "Peu nombreuses sont les personnalités créatrices qui mettent en mouvement une civilisation et l'entraînent en avant". Et la croissance d'une civilisation se mesure par la montée en autonomie et en créativité de la société. Le progrès d'une civilisation ne peut donc s'identifier au seul progrès technique, car les défis les plus sévères sont bien souvent des défis intérieurs concernant par exemple l'organisation de la société ellemême. Tel est aujourd'hui, selon Toynbee, le cas pour la civilisation occidentale.

\section{2-3) Déclin d'une civilisation}

Pour Toynbee, "les déclins ne sont ni inévitables ni irréparables, mais si le processus de désintégration est à même de se poursuivre, je découvre qu'il suit apparemment un modèle commun dans la plupart des cas". A l'origine, se trouve le renversement pervers du mécanisme de la mimésis (ou imitation) qui s'est instauré entre les élites et le peuple. Ce mécanisme présente un double aspect contradictoire :

Phase de CReation

Répand ses inventions

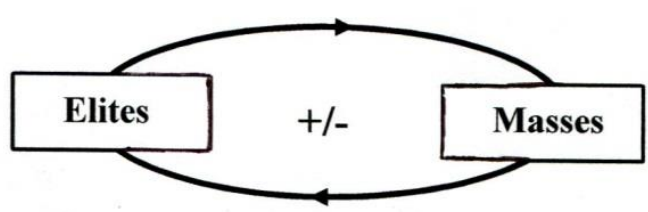

Imitation
Phase de declin

Impose ses règles

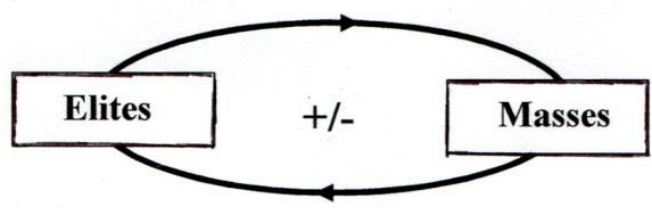

Mécanisation 
- pour créer, la minorité créatrice doit répandre et imposer ses inventions et ses valeurs, ce qu'elle réussit grâce au processus d'imitation (la majorité imitant la minorité créatrice) ${ }^{10}$. C'est la phase de création.

- mais le mécanisme peut se pervertir lorsque les élites ayant perdu tout pouvoir créateur restent hypnotisées par le dressage qu'elles ont inculqué à une masse soumise (ou "prolétariat intérieur"). C'est la phase du déclin.

Dans cette seconde phase, la mimésis s'est généralisée à l'ensemble de la civilisation. Il n'y a plus nouveauté, adaptation, mais répétition mécanique du passé. La tradition cesse d'être rappel stimulant à l'élan créateur pour se cristalliser en répétition idolâtrique d'un passé éphémère, d'une institution, d'une règle, d'un outil. Cette idolâtrie peut revêtir deux formes :

- une forme passive (koros : "s'endormir sur ses lauriers"). C'est l'assoupissement après une phase de gloire et de succès. Pourquoi un groupe ou un individu cessent-ils de créer, phénomène pourtant des plus communs ? Il semble qu'un groupe ne puisse pas relever plus d'un défi à la fois. Après ce "tour de force", il s'endort sur son succès. "Le créateur qui a connu une fois le succès est gravement handicapé, de par son succès même, lorsqu'il tente de reprendre son rôle créateur" écrit Toynbee.

- une forme active (hybris: se précipiter dans la catastrophe ou até en grec). C'est en quelque sorte Gribouille se jetant dans l'eau pour ne pas être mouillé. Ce comportement, également très fréquent dans l'histoire, exprime la démesure et la répétition mécanique de ce qui a réussi dans le passé mais qui justement ne peut qu'anticiper l'échec

Des nombreux exemples où se joue la tragédie koros-hybris-até, Toynbee va extraire à titre illustratif :

- pour l'idolâtrie d'un passé éphémère : Athènes qui s'aveugle sur son rôle transitoire "d'éducatrice de la Grèce", la République de Venise incapable de prendre la tête du mouvement pour l'unité italienne réalisée par un petit Etat périphérique (le Piémont),

- pour l'idolâtrie d'une institution : l'empire romain d'Orient qui conduit en 1000 ans la chrétienté orthodoxe à sa perte, celle-ci n'ayant de cesse que de vouloir le ressusciter,

- pour l'idolâtrie d'un outil ou d'une technique : pratiquement toute l'histoire de l'art de la guerre depuis la phalange macédonienne jusqu'à l'emploi de l'arme blindée en 1939.

\section{2-4) Pour conclure}

Si l'histoire des sociétés humaines n'est rien d'autre, sur un plan socio-culturel, que le prolongement de l'évolution du vivant, comme le pense Teilhard de Chardin, alors le modèle de Toynbee vient lui aussi en contestation de la théorie synthétique de l'évolution (ou modèle standard). Si le Défi semble s'apparenter, dans un premier temps, à la Nécessité de Monod, il s'agit en effet d'une notion plus large que la seule sélection naturelle. Quant à la Réponse, elle, est beaucoup plus qu'un simple produit du Hasard, même si elle inclut ce dernier dans son processus d'élaboration par tâtonnement. Comme le souligne Toynbee, la Réponse présuppose l'intelligence finalisée et libre de l'acteur humain, l'invention créatrice qui imagine les solutions possibles ; c'est-à-dire la pensée abductive de la systémique, pensée qui ne peut se réduire à l'induction et encore moins à la déduction.

Les comportements intelligents et finalisés que nous observons dans l'Histoire ne seraient ainsi rien d'autre que le prolongement hypertrophié de comportements déjà finalisés d'invention, de type symbiotique par exemple, déjà présents dans l'évolution du vivant. Ainsi, depuis le big-bang jusqu'aux civilisations humaines, une même loi d'évolution serait bien en œuvre dans l'Univers, une loi agissant au travers de processus de nature certes différents (biologiques puis socio-culturels), mais analogues sur le plan formel.

\footnotetext{
${ }^{10}$ On pourrait rapprocher ici l'analyse de Toynbee de la thèse du désir mimétique de René Girard.
} 


\section{L'Evolution et l'Histoire sont-elles orientées ?}

Constater que les systèmes sociaux comme les systèmes vivants sont finalisés et capables de transmettre cette propriété à leurs descendants ne dit rien quant à la source de cette finalité. Ne faut-il pas alors reconnaître que cette finalité est inhérente à la nature ellemême, à son inventivité qui sait faire feu de tout bois, notamment en utilisant la contingence et le hasard pour arriver à ses fins ? Peut-on voir, comme le pensait le grand naturaliste Pierre Grassé $^{11}$, une telle finalité à l'œuvre au niveau de la "Grande Evolution", celle qui fait émerger de nouveaux types d'organisation du vivant comme les vertébrés, les mammifères ou les hominidés? Par quels mystérieux processus des molécules organiques se sont-elles assemblées entre elles, il y a près de quatre milliards d'années, pour donner naissance à la vie ? Comment a pu se développer, pour devenir le support de la pensée et de la conscience, ce monument de complexité qu'est le cerveau humain aux cent milliards de neurones, chacun interconnecté en moyenne dix mille fois avec les autres ? Comment ont pu surgir le langage symbolique humain puis se développer les civilisations?

Toutes ces questions mettent au défi les scientifiques de penser des phénomènes où interagissent une multitude de facteurs, où se combinent des principes de régulation et de déséquilibre, où se mêlent contingence et déterminisme, création et destruction, ordre et désordre, où prolifèrent des systèmes à l'architecture de plus en plus élaborée. De l'atome au cerveau humain puis à la société interconnectée et mondialisée, on pressent une fabuleuse montée de complexité. Une complexité qui résulte d'abord de la richesse et de la diversité des relations ou connexions, elles-mêmes structurées selon des architectures ordonnées et régulières. C'est pourquoi, pour reprendre l'exemple du cerveau, un ensemble gazeux de cent milliards d'atomes ne présentera jamais des caractéristiques comparables à celui-ci.

Dans l'évolution du cosmos on passe ainsi de systèmes simples (la particule élémentaire, l'atome) à des systèmes de plus en plus fortement organisés (la bactérie, la cellule eucaryote, la plante, l'animal, l'homme, la société) dont on peut, le plus souvent, repérer l'architecture interne à base de diversité et de redondance, c'est-à-dire de répétition d'un même motif comme il en va dans les structures fractales. Ces systèmes organisés s'opposent aux agrégats (un tas de sable, un nuage, une étoile, une foule...) lesquels, bien que comprenant un grand nombre d'éléments, ne sont que faiblement organisés.

Parce qu'il n'y a pas d'organisation sans redondance, la réduction de la variété sera donc, dans un premier temps, l'effet habituel de l'organisation. Ainsi, l'ordre répétitif du cristal fige-t-il le corps en fusion, les molécules perdant la liberté de placement dont elles disposaient à l'état liquide. Mais dans un second temps, l'organisation fait apparaitre des possibilités relationnelles nouvelles entre sous-ensembles stabilisés d'éléments que l'on appelle alors des sous-systèmes. Ainsi, va-t-on rencontrer des relations entre les soussystèmes eux-mêmes ou entre un sous-système particulier et certains composants de rang inférieur, ce qui introduit la notion de niveaux d'organisation. Cet accroissement de la richesse relationnelle permet à son tour de faire émerger dans le système des configurations nouvelles ... d'où une augmentation correspondante de la variété et ainsi de suite.

Lorsque ce second effet l'emporte sur le premier, la complexité du système tend à augmenter. On dit alors que l'on a affaire à une organisation complexifiante et on parle de complexification. Dans l'évolution du vivant, puis dans l'évolution sociale et culturelle, c'est bien de cela qu'il s'agit. Au cours des temps géologiques puis historiques, apparaissent des systèmes de plus en plus complexes. Ceci est mis en évidence par la figure ci-après, laquelle superpose aux deux infinis de Pascal (l'infiniment petit et l'infiniment grand) un troisième infini, l'infiniment complexe, orienté dans le sens de la flèche du temps de l'évolution. A l'échelle des temps géologiques puis historiques, dans l'intervalle d'espace séparant

\footnotetext{
${ }^{11}$ Pierre Grassé, L'évolution du vivant, Albin Michel 1973
} 
l'infiniment petit (la particule élémentaire au diamètre estimé de $10^{-15}$ mètres) de l'infiniment grand (l'ensemble des galaxies au diamètre supposé de $10^{26}$ mètres) se déploie ainsi un gigantesque phénomène de complexification.

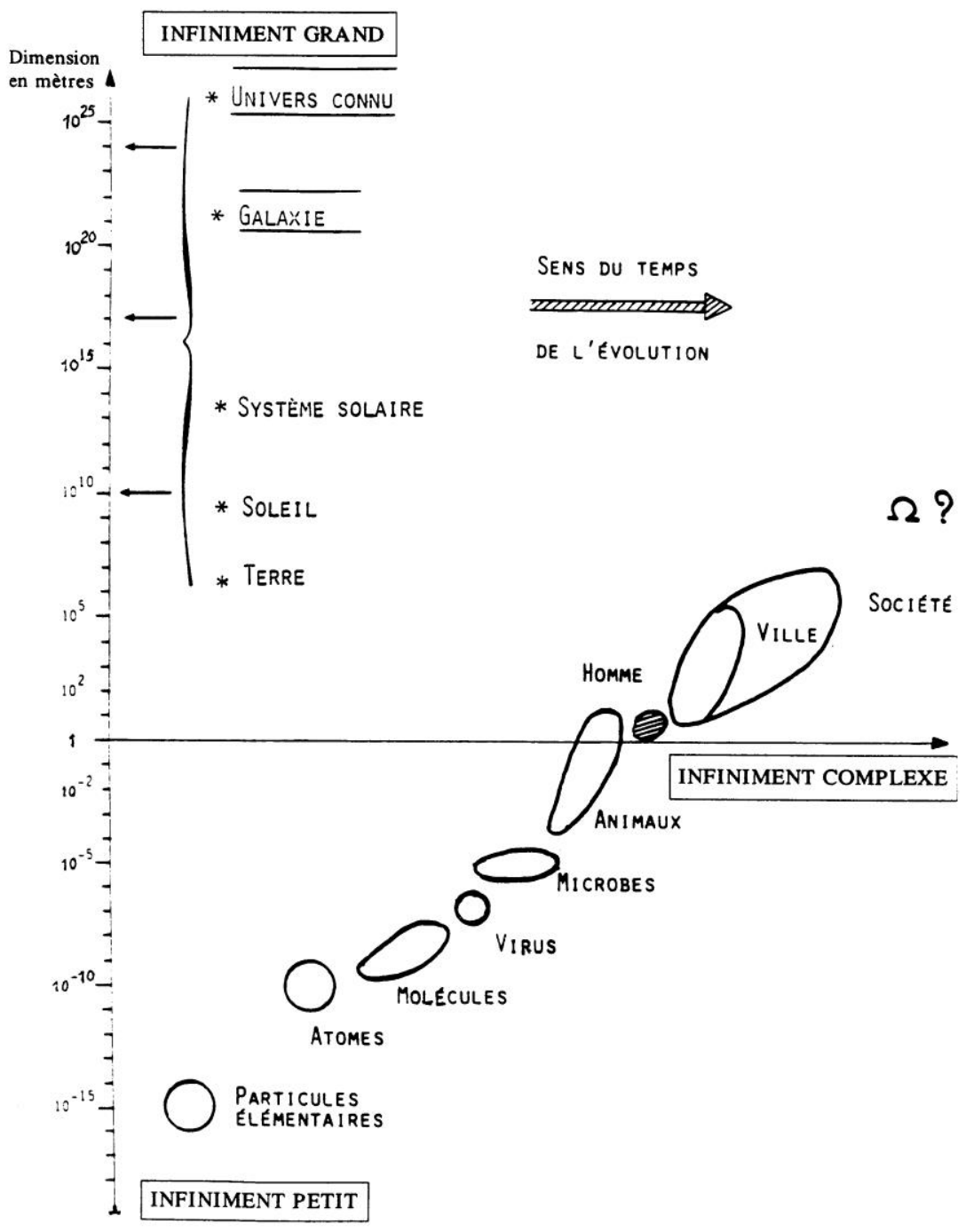

Ce troisième infini avait été décrit dès les années 1930 par Pierre Teilhard de Chardin $^{12}$ sous le nom de loi de complexification ou de complexité croissante. A cette première loi, Teilhard en ajoutait une seconde, la loi de complexité-conscience, signifiant parlà que l'hyper complexité s'accompagne, à partir d'un certain seuil, de l'apparition de psychismes de plus en plus riches, c'est-à-dire de conscience ou d'esprit. Et cet esprit devient à son tour un facteur supplémentaire de variété dans le jeu relationnel... d'où de nouvelles possibilités d'accroissement de la complexité.

A ce stade de la réflexion, deux remarques complémentaires peuvent être faites :

1. A mi-distance de l'infiniment grand et de l'infiniment petit, l'homme et ses créations (les systèmes économiques, politiques, sociaux, culturels) occupent une place de choix dans la montée en complexité. En front de vague de la complexification, ils constituent en quelque sorte la tête chercheuse de l'évolution. La culture, l'histoire, "l'esprit" se trouvent ainsi réinsérés, selon le souhait d'Edgar Morin, dans l'ordre des réalités naturelles.

\footnotetext{
${ }^{12}$ TEILHARD DE CHARDIN P. : Le phénomène humain, Seuil 1955
} 
2. Le temps de l'évolution complexifiante coule en sens inverse de celui de la physique. Depuis le célèbre second principe de la thermodynamique nous savons en effet que l'évolution d'un système fermé ne peut se faire que vers la disparition de son organisation interne (équiprobabilité des configurations) repérée par la croissance de l'entropie. Au temps entropique s'opposerait ainsi le temps de l'évolution ou temps néguentropique.

La contradiction entre ces deux temporalités n'est toutefois qu'apparente. Considéré restrictivement, le temps entropique concerne des systèmes fermés alors que le temps de l'évolution s'observe uniquement sur les systèmes ouverts. Or, tous les systèmes complexes sont ouverts ! Dans un univers où l'entropie est nécessairement croissante peuvent ainsi exister - pour un temps donné - des foyers locaux de complexification, ceci pour autant que ces foyers empruntent de l'énergie, de la matière et de l'information à leur environnement. Un îlot de complexité au milieu du grand fleuve entropique, telle serait en définitive la situation de notre Terre et des phénomènes biologiques et sociétaux qui continuent de s'y dérouler.

Ainsi, l'évolution "travaille" selon un "tâtonnement orienté", écrit Teilhard, tâtonnement observé également dans l'Histoire, à l'occasion des diverses inventions (techniques, organisationnelles, symboliques, etc.) faites par l'homme - où l'on parle alors de processus d'abduction - inventions qui conduisent à une montée en complexité et en richesses intellectuelles des sociétés et des cultures. Tout se passe comme si ce tâtonnement était orienté, en quelque sorte, par un grand attracteur que Teilhard de Chardin appelle le Point Oméga et qui donne à la "grande évolution" sa finalité.

Qu'une telle finalité soit présente dans la nature, c'est ce qu'après Teilhard, ont reconnu honnêtement bien d'autres scientifiques, comme le biologiste et prix Nobel Christian de Duve se déclarant par ailleurs agnostique : "Pour moi, l'Univers est ainsi fait qu'il doit donner naissance à la vie, probablement en de nombreux endroits. Et la vie est ainsi faite qu'elle doit, du moins en un certain nombre de cas, donner naissance à la pensée...Il suffit, pour s'en rendre compte, de regarder les mécanismes remarquables qui sous-tendent le fonctionnement des êtres vivants, de contempler l'extraordinaire diversité d'organismes que l'évolution biologique a produite, de songer à toutes les formes de culture que la pensée humaine a déjà générées et qu'elle générera sans doute dans l'avenir". Ou encore le paléoanthropologue Yves Coppens ${ }^{13}$, codécouvreur de l'australopithèque Lucy : "La matière de l'Univers se complique et s'organise dès qu'elle existe, elle se complique et s'organise davantage lorsqu'elle devient vivante,... elle se complique encore plus et s'organise encore mieux lorsqu'une partie de cette matière vivante devient pensante... L'histoire de notre Univers a donc un sens, une direction et en même temps du sens".

Et que cette finalité soit également présente dans l'histoire humaine, c'est ce qu'ont pensé aussi nombre de philosophes qui ont réfléchi sur un éventuel sens de l'Histoire depuis le siècle des Lumières. On rappellera pour mémoire Georg Hegel (1770-1831) voyant dans l'Histoire l'avènement de la Raison universelle ou Esprit absolu, Karl Marx (1818-1883) y voyant au contraire l'avènement d'une société enfin libérée de toutes les oppressions économiques et politiques et plus près de nous, du philosophe chrétien Jacques Maritain (1882-1973) y discernant la possibilité ${ }^{14}$ d'un progrès de la conscience morale et d'une accession des peuples à la majorité politique et sociale. Sous des expressions certes très différentes, ces points de vue de philosophes quant au "sens de l'Histoire" ne sont pas contradictoires avec les deux premiers articles du credo de Teilhard de Chardin ${ }^{15}$ : "Je crois que l'Univers est une Evolution. Je crois que l'Evolution va vers l'Esprit."

\footnotetext{
${ }^{13}$ Yves COPPENS, préface à l'ouvrage de André DANZIN et Jacques MASUREL, Teilhard de Chardin, visionnaire d'un monde nouveau, Editions du Rocher, 2005, p.8

${ }_{14}^{14}$ Jacques MARITAIN, Pour une philosophie de l'Histoire, Seuil, 1959

${ }^{15}$ Pierre TeILHARd DE CHARdin, Comment je crois, Tome 10 des Euvres complètes, Seuil, 1969, p.117
} 
